\title{
Adrénoleucodystrophie : progrès récents et perspectives thérapeutiques
}

L'adrénoleucodystrophie liée au chromosome $\mathbf{X}$ est une maladie peroxysomiale de la $\beta$-oxydation des acides gras à très longue chaîne qui s'accumulent dans la substance blanche du système nerveux central et dans d'autres tissus, dont la surrénale. La nature exacte de l'enzyme déficiente n'est pas complètement connue à ce jour. Il n'existe aucune relation constante entre l'anomalie biochimique et l'intensité des manifestations cliniques qui vont des formes mortelles avant cinq ans dans un grand syndrome neurologique, aux formes pratiquement asymptomatiques; l'intervention d'une réaction immune contre des tissus nerveux dans lesquels se sont accumulés des lipides anormaux a été évoquée. La proximité génétique du locus de l'adrénoleucodystrophie et de celui de la vision des couleurs, associée à la fréquente association entre le daltonisme et cette maladie, suggère que les deux loci pourraient être modifiés par un événement commun de réarrangement d'ADN.

\section{Patrick Aubourg}

\section{ADRESSE}

P. Aubourg: chargé de recherche à l'Inserm. Inserm U. 188, hôpital Saint-Vincent-dePaul, 74, avenue Denfert-Rochereau, 75014 Paris Cedex, France.

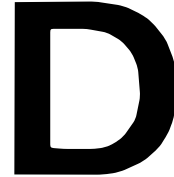

epuis la reconnaissance du rôle du peroxysome en pathologie humaine, l'adrénoleucodystrophie et les autres maladies apparentées ont fait l'objet, au cours des dix dernières années, d'une recherche qui a amené des progrès réellement importants dans la compréhension de ces maladies. Nous discuterons, ici, essentiellement de l'adrénoleucodystrophie liée à l'X (ALD-X) et présenterons les développements récents qui concernent les aspects phénotypiques, les méthodes de diagnostic, le déficit enzymatique, et les aspects génétiques et thérapeutiques. Il est aujourd'hui bien établi que les ALD néonatales constituent une entité totalement différente de l'ALD-X. Elles se transmettent selon le mode autosomique récessif et sont la consé- quence, comme le syndrome de Zellweger, d'un déficit pluri-enzymatique en rapport avec une anomalie de la translocation des enzymes peroxysomiales à travers la membrane du peroxysome. L'ALD-X est considérée, en revanche, comme une maladie peroxysomiale uni-enzymatique, sans anomalie morphologique des peroxysomes [1].

\section{Aspects phénotypiques}

En 1923, Simerling et Creutzfeldt [2] rapportèrent le cas d'un petit garçon âgé de sept ans, qui allait bien jusqu'à l'âge de trois à quatre ans lorsque fut noté, pour la première fois, une mélanodermie. A l'âge de six ans et demi, il commença à présenter des troubles du comportement, des anomalies du langage et une instabilité de la marche. En quelques mois, il 


\section{RÉFÉRENCES}

1. Poll The BT, Vameca J, Draye JP, Saudubray JM. Un nouveau groupe d'erreurs innées du métabolisme: les maladies peroxysomiales. médecine/sciences 1988 ; 4 : 553-9.

2. Siemerling E, Creutzfeldt HG. Bronzekrankheit und sklerosierende encephalomyelitis (diffuse sclerose). Arch Psychiatr 1923 ; 68 : 217-44.

3. Moser HW, Moser AB, Singh I. O'Neill BP. Adrenoleukodystrophy: Survey of 303 cases: biochemistry, diagnosis and therapy. Ann Neurol $1984 ; 16: 628-41$.

4. Griffin JW, Goren E, Schaumburg H, et al. Adrenomyeloneuropathy: A probable variant of adrenoleukodystrophy. Neurology 1977 ; 27 : 1107-13.

5. Powers JM, Shaumburg $\mathrm{HH}$. The adrenal cortex in adrenoleukodystrophy. Arch Pathol 1973 ; 96 : 305-10.

6. Singh I, Moser AB, Moser HW, Kishimoto Y. Adrenoleukodystrophy: Impaired oxidation of very long chain fatty acids in white blood cells, cultured skin fibroblasts and amniocytes. Pediatr Res 1984 ; 18 : 286-9.

7. Hashimoto $T$. Individual peroxysomal beta-oxidation enzymes. Ann NY Acad Sci $1982 ; 386: 5-12$.

8. Chen WW, Watkins PA, Osumi T, Hashimoto T, Moser HW. Peroxisomal beta-oxidation enzymes in adrenoleukodystrophy. Proc Natl Acad Sci USA 1987 ; 84 : 1425-8.

9. Hashmi M, Stanley W, Singh I. Lignoceroyl-CoASH ligase: Enzyme defect in fatty acid beta-oxidation system in X-linked childhood adrenoleukodystrophy. FEBS Lett 1986 ; $196: 247-50$.

10. Wanders RJA, Van Roermund CWT, Van Wyland MJA, et al. X-linked Adrenoleukodystrophy: identification of the primary defect at the level of a deficient peroxisomal very long chain fatty acyl-CoA synthetase using a newly developed method for the isolation of peroxisomes from skin fibroblasts. J Inher Metab Dis 1988 ; 11 (Suppl. 2) :

devint spastique*, puis incapable de marcher, d'avaler et décéda à l'âge de sept ans. Ce tableau clinique, qui correspond à la forme cérébrale infantile de l'ALD-X, la plus fréquemment observée (40\%), ne représente qu'une des formes cliniques de cette maladie [3]. En effet, la spécificité d'un test diagnostique pour l'ALD-X a permis de démontrer le caractère extrêmement polymorphe de celle-ci, les différents phénotypes cliniques pouvant être observés au sein d'une même famille. Ceux-ci ainsi que leur fréquence relative sont présentés dans le Tableau I. Les formes les plus fréquentes, cérébrales infantiles ou juvéniles, débutent dans $85 \%$ des cas par des signes neurologiques : troubles du comportement et difficultés intellectuelles, surdité et diminution de l'acuité visuelle. Approximativement $30 \%$ des patients présentent des crises convulsives qui peuvent être inaugurales. Plus de $85 \%$ des patients ont une diminution de la réponse du cortisol plasmatique à l'ACTH, ce qui reflète une insuffisance surrénale. Celle-ci est souvent limitée cliniquement à une mélanodermie, mais une insuffisance surrénale aiguë peut révéler l'ALD-X dans $15 \%$ des cas. Une fois que les signes neurologiques deviennent évidents, l'évolution est souvent rapide, avec apparition de signes pyramidaux et cérébelleux, entraînant un état végétatif en deux ans. L'enfant est alors incapable de se mouvoir, de parler, de voir et de s'alimenter. Il peut rester dans cet état plusieurs années, mais le décès survient en règle avant l'âge de cinq ans. Le CT-Scan** ou l'IRM ${ }^{* * *}$ (figure 1) montrent des lésions de démyélinisation qui prédominent dans les lobes pariétaux et occipitaux.

L'adrénomyéloneuropathie (AMN) débute habituellement entre 20 et 30 ans, chez un homme jusque-là en bonne santé, par des troubles de l'équilibre, une raideur des jambes et des signes d'insuffisance surrénale[4]. L'atteinte neurologique est lentement progressive en cinq à 15 ans. La sévérité des troubles de l'équilibre nécessite alors souvent l'utilisation d'une canne ou d'une chaise roulante. L'examen neurologique révèle une paraplégie spastique et une atteinte des cordons postérieurs de la moelle. La vitesse de conduction motrice des nerfs périphériques peut être réduite. Les fonctions intellectuelles sont préservées quoiqu'une étude neuropsychologique détaillée puisse mettre souvent en évidence des déficits cognitifs. Alors que le CT-scan reste normal, l'IRM montre dans environ $30 \%$ des cas des anomalies de la substance blanche cérébrale. Les formes adultes cérébrales ressemblent à une schizophrénie ou une démence, avec ou sans signes neurologiques. Les fonctions surrénaliennes peuvent rester normales et l'atteinte de la substance blanche démontrée par le CT-scan

\footnotetext{
* Hypertonie spastique: augmentation du tonus musculaire par exagération du réflexe monosynaptique.

** Tomodensitométrie ou scannographie. ** Imagerie par résonance magnétique.
}

\begin{tabular}{|lc|}
\hline \multicolumn{2}{|c|}{$\begin{array}{c}\text { Tableau I } \\
\text { PHÉNOTYPE DE L'ADRÉNOLEUCODYSTROPHIE LIÉE A L'X }\end{array}$} \\
\hline 1. ALD infantile (<10 ans) & $40 \%$ \\
2. ALD juvénile (10-20 ans) & $10 \%$ \\
3. ALD : forme adulte cérébrale & $3 \%$ \\
4. Adrénomyéloneuropathie & $25 \%$ \\
5. Addison sans atteinte neurologique & $10 \%$ \\
6. Pré- et asymptomatique & $12 \%$ \\
\hline
\end{tabular}


ou l'IRM est souvent le seul élément qui puisse orienter vers une ALD-X avant que celle-ci soit confirmée par les tests biochimiques. Comme on l'a $\mathrm{vu}$, l'insuffisance surrénale accompagne les différents modes de présentation neurologique de l'ALD-X. Elle peut résumer à elle seule, l'expression de la maladie.
Au moins $20 \%$ des femmes hétérozygotes présentent des signes neurologiques qui ressemblent à ceux observés dans l'AMN. Le diagnostic le plus souvent évoqué est celui de sclérose en plaques qui n'est remis en question que si on identifie un sujet mâle atteint de l'une des formes d'ALD-X dans la famille.

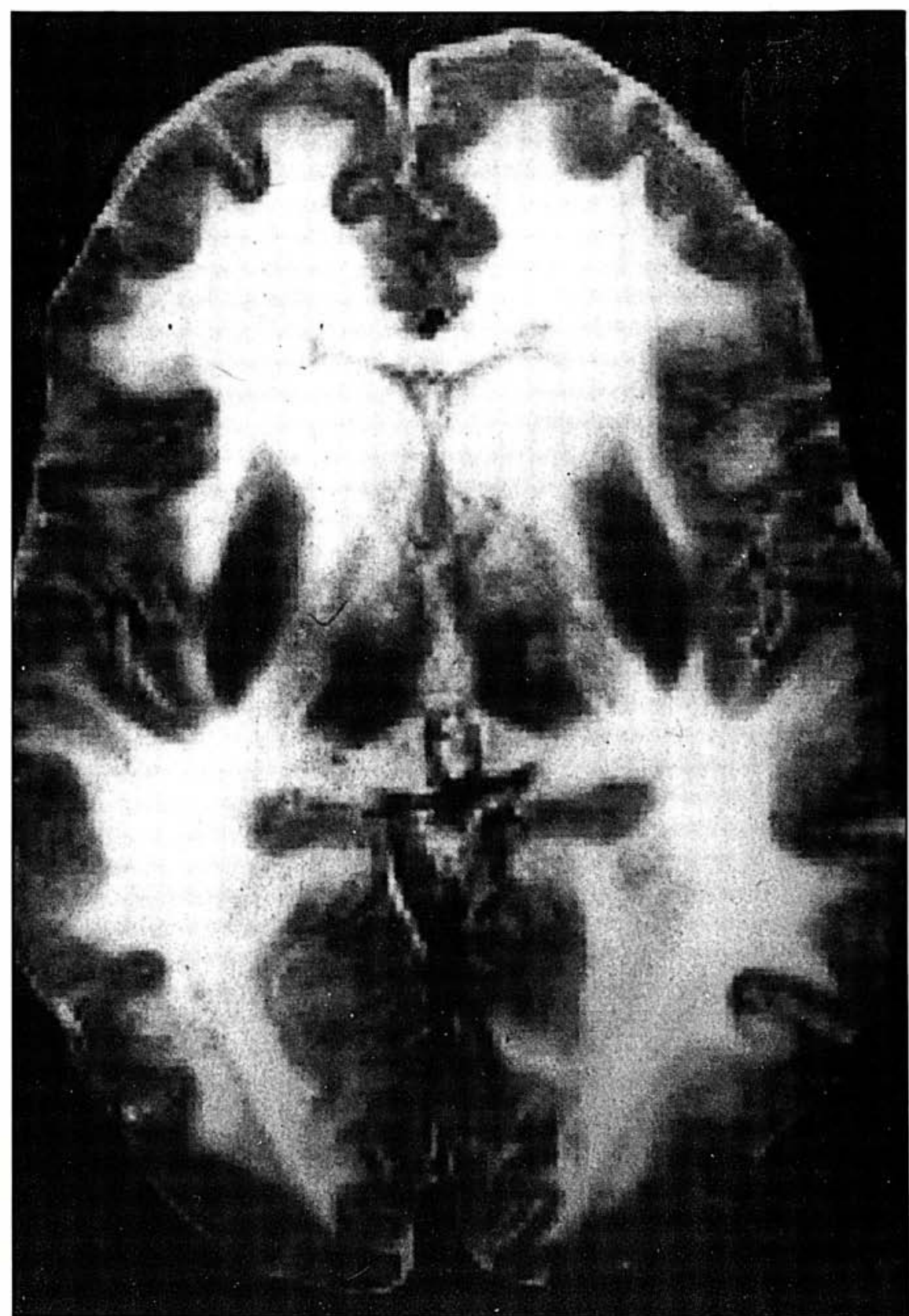

Figure 1. Image de résonance magnétique nucléaire (séquence pondérée en T2) montrant un signal intense anormal de toute la substance blanche chez un jeune patient dont la maladie évolue depuis huit mois.
L'étude systématique des différents membres d'une même famille à partir du cas index a permis de démontrer que certaines personnes présentaient l'anomalie biochimique de l'ALD-X sans aucune manifestation clinique. Ainsi, des hommes âgés de plus de 60 ans peuvent très bien ne présenter aucun signe clinique.

Cette variabilité de l'expression clinique (il ne s'agit pas de manifestations alléliques différentes) pose des problèmes pratiques évidents. En effet, malgré la fiabilité du diagnostic biochimique, celui-ci ne permet pas de différencier les différentes formes cliniques et donc lorsque, dans une famille, on détecte qu'un petit garçon de quatre ans est atteint biochimiquement de cette maladie, rien ne permet de prévoir s'il présentera une forme cérébrale grave, une forme adulte, voire s'il restera totalement asymptomatique. Cette variation phénotypique suggère également que le déficit enzymatique est nécessaire mais non suffisant pour que s'exprime la maladie. Ces différents aspects seront discutés plus loin.

\section{L'accumulation d'acides gras à très longue chaîne}

Utilisation pour le diagnostic et la détection des hétérozygotes. Une étape importante dans la connaissance de l'ALD-X a été l'observation faite par Powers et Schaumburg [5] que les cellules du cortex surrénalien avaient un aspect strié en microscopie électronique. Ces cellules, ainsi que les macrophages du système nerveux central (SNC), contiennent des inclusions électrodenses ayant un aspect de fente. Des études morphologiques et biochimiques ont permis d'établir qu'elles étaient constituées de cholestérol estérifié avec des acides gras saturés ayant une longueur de chaîne allant de C24 à C30. L'acide hexacosanoïque (C26:0)* et l'acide pentacosanoïque (C25:0) constituent entre 30 et $60 \%$ des acides gras des fractions gangliosides et des esters du cholestérol de la substance blanche du SNC des

* (C26:0): acide gras à 26 atomes de carbone et dépourvu de double liaison. 
patients atteints d'ALD-X, alors qu'ils ne sont pratiquement pas retrouvés dans ces fractions lipidiques chez les sujets normaux. Cette accumulation d'acides gras à très longue chaîne (AGTLC) a depuis été retrouvée dans d'autres maladies peroxysomiales (syndrome de Zellweger, ALD néonatale et Refsum infantile) mais en association avec d'autres anomalies biochimiques [1]. Leur quantification dans le plasma, les globules rouges ou les fibroblastes permet avec certitude d'identifier les sujets atteints, même si ici leur accumulation reste plus modeste $(0,08 \pm 0,02 \%$ des acides gras totaux pour le C26:0 contre 0,014 $\pm 0,010 \%$ chez les sujets normaux dans le plasma). La détection des femmes hétérozygotes est possible dans environ $85 \%$ des cas. Couplée à cette méthode biochimique, l'analyse de l'ADN à l'aide de la sonde anonyme Stl4/DXS52 (voir plus loin) permet d'améliorer encore la précision de cette détection. Le diagnostic anténatal repose sur la démonstration d'une élévation des AGTLCdans le liquide amniotique ou les cellules trophoblastiques. Comme on le verra plus loin, cette accumulation d'AGTLC résulte d'un trouble de leur $\beta$-oxydation et l'étude quantitative des AGTLC peut ainsi être couplée dans la mesure de leur oxydation avec les tissus et ce, plus particulièrement pour le diagnostic anténatal. Il n'y a cependant aucune corrélation entre le phénotype clinique et le taux des AGTLC observé. Le diagnostic anténatal permet donc de détecter un foetus à risque mais ne permet pas de déterminer quel phénotype il présentera (forme cérébrale, AMN, forme cliniquement asymptomatique).

\section{Le déficit enzymatique}

I. Singh [6] établit pour la première fois en 1984 que l'accumulation des AGTLC résultait d'un trouble de leur $\beta$-oxydation. Il étudia l'oxydation de différents acides gras dont la longueur de chaîne variait du $\mathrm{Cl} 6$ au C26 et démontra que dans les cellules de patients atteints d'ALD-X, la dégradation de l'acide lignocérique (C24:0) et du C26:0 était réduite à 15 et $20 \%$ respectivement des valeurs normales, tandis que l'oxydation de

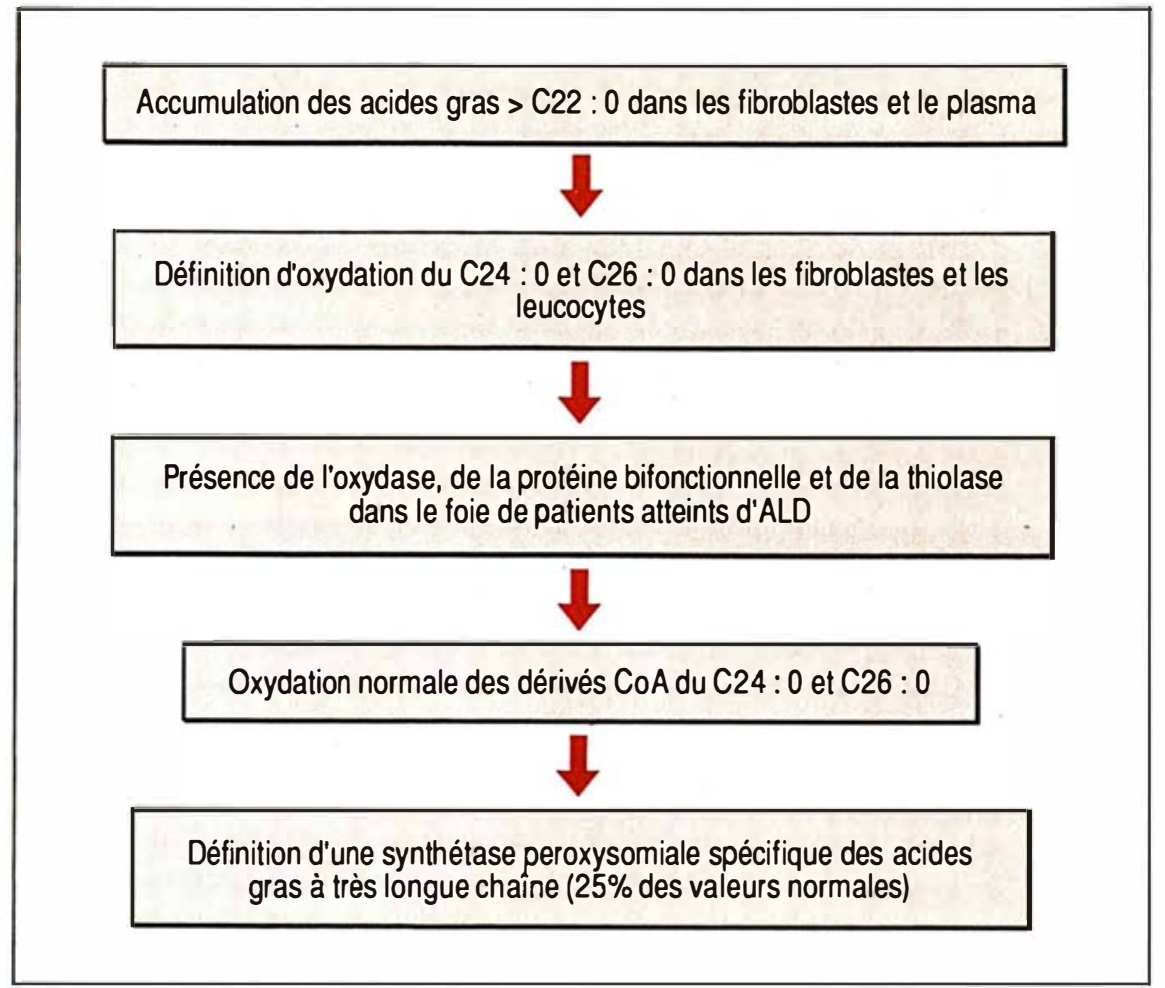

Figure 2. Principales étapes ayant permis d'approcher le déficit biochimique de l'adrénoleucodystrophie liée à I'X.

l'acide stéarique (Cl8:0) était normale. A peu près à la même époque, différentes études montrèrent que les acides gras de longueur de chaîne inférieure au Cl8 étaient métabolisés principalement par la voie bien connue de la $\beta$-oxydation mitochondriale, alors que les acides gras de longueur de chaîne supérieure étaient catabolisés dans le peroxysome. Ces observations permirent de suggérer pour la première fois que l'ALD-X pouvait être une maladie peroxysomiale (figures 2 et 3 ).

Le système de la $\beta$-oxydation peroxysomiale a été particulièrement étudié par Hashimoto et al. [7] et les trois dernières enzymes ont été purifiées et clonées chez le rat (figure 3). Initialement, l'attention s'est donc portée, tout naturellement, sur ces trois enzymes: l'oxydase, la protéine bifonctionnelle et la thiolase. En effet, il n'existe pas de moyens biochimiques capables d'étudier séparément et de manière fiable les diffé- rentes étapes de la $\beta$-oxydation peroxysomiale. Trois types de résultats ont permis d'affirmer que ces enzymes n'étaient pas déficitaires dans l'ALD-X : (1) l'utilisation d'anticorps polyclonaux dirigés contre chacune de ces trois enzymes chez le rat et réagissant contre leurs homologues humaines a permis de démontrer que ces trois enzymes étaient présentes dans le foie et les fibroblastes de patients atteints d'ALD$\mathrm{X}$ [8]. (2) Les gènes et $\mathrm{ADN}$ complémentaires de ces trois enzymes ayant été clonés chez le rat, il a été possible de démontrer, en étudiant des cellules hybrides contenant une partie du chromosome $\mathrm{X}$, que les gènes codant pour ces enzymes n'étaient pas situés sur le chromosome $\mathrm{X}$ et en particulier sur la portion distale du chromosome $\mathrm{X}$ où avait été localisée cet te affection génétique (P. Aubourg et al., résultats non publiés). (3) Enfin, Haschmi et al. [9] remarquèrent les premiers que 
la dégradation du dérivé CoA de l'acide lignocérique (C24:0) se faisait normalement dans les fibroblastes de patients atteints d'ALD-X. Cette observation, confirmée depuis par d'autres équipes, suggère donc que le défaut d'oxydation des AGTLC dans l'ALD-X résulte d'un déficit au niveau de l'enzyme qui catalyse la première réaction (figure 3), à savoir une synthétase (ou ligase). La mesure directe de l'activité de cette synthétase spécifique des AGTLC n'a montré initialement qu'une réduction de l'ordre de $50 \%$. En fait, il existe une synthétase des AGTLC à la fois dans le peroxysome et les microsomes. Il a fallu attendre des développements

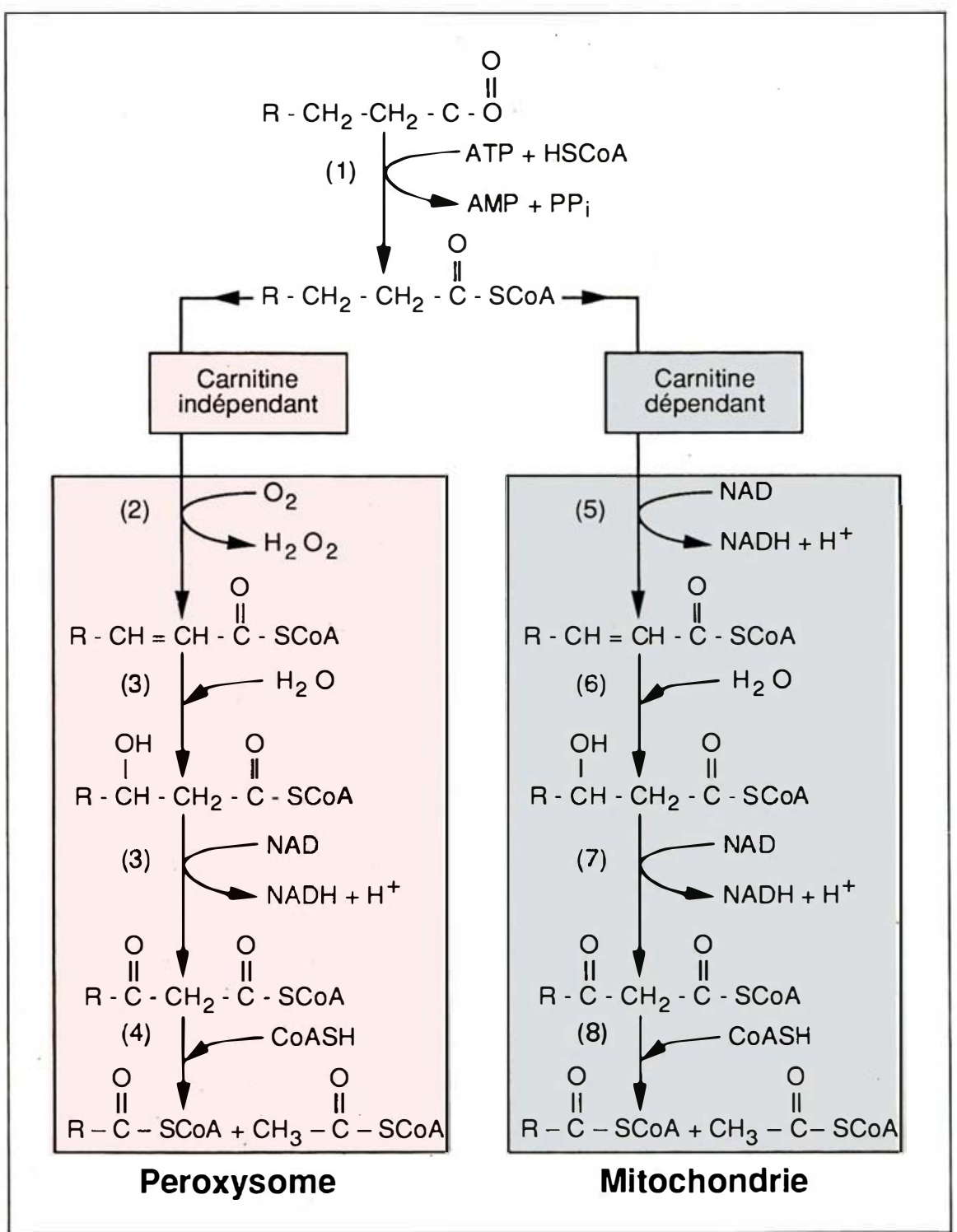

Figure 3. Comparaison des systèmes de $\beta$-oxydation peroxysomiale et mitochondriale des acides gras. (1) Acyl-CoA synthétases peroxysomiale et mitochondriale; (2) Acyl-CoA oxydase; (3) Protéine bifonctionnelle (énoyl-CoA hydratase/3-hydroxyacyl-CoA déhydrogénase; (4) $\beta$-cétothiolase; (5) Acyl-CoA déhydrogénase; (6) Enoyl-CoA hydratase; (7) 3-hydroxyacyl-CoA déhydrogénase; (8) $\beta$-cétothiolase. Les acides gras de longueur de chaine supérieure en C22 sont préférentiellement oxydés dans le peroxysome.

récents permettant une meilleure séparation des fractions mitochondriales et peroxysomiales pour pouvoir réellement démontrer qu'il existait bien une diminution significative de la synthétase peroxysomiale des AGTLC dans les fibroblastes de patients atteints d'ALDX [10]. Quoique le déficit observé soit du même ordre de grandeur que celui de la $\beta$-oxydation peroxysomiale ( 15 à $20 \%$ ), il n'est pas encore établi si le déficit concerne l'enzyme elle-même ou une protéine activatrice. L'enzyme n'a pas encore été purifiée et, malgré des progrès importants dans le fractionnement cellulaire, sa purification s'avère difficile. Si la synthétase peroxysomiale spécifique des AGTLC a, comme c'est probablement le cas, une grande similitude avec ses homologues mitochondriales et microsomiales, il sera probablement plus simple, dans un premier temps, de purifier puis de cloner le gène d'une autre synthétase et, dans un deuxième temps, en utilisant l'ADN complémentaire de l'une de ces synthétases, de cribler une librairie hépatique ou cérébrale en utilisant des conditions de stringence différente.

\section{Une autre approche du déficit moléculaire : la génétique réverse}

L'étude initiale permettant de localiser le gène de l'ALD-X a été réalisée chez trois familles noires américaines : les femmes étaient hétérozygotes à la fois pour l'ALD-X et une variante électrophorétique de la glucose-6-phosphate déshydrogénase (G6PD). Le gène de la G6PD avait été antérieurement localisé sur la portion distale du bras long du chromosome X (Xq2-8). Cette étude initiale a été poursuivie avec l'utilisation d'une sonde ADN anonyme (Stl4/DXS52) localisée dans la même région. Cette sonde met en évidence un polymorphisme des fragments d'ADN coupés par des enzymes de restriction Taq-I ou Msp-I et analysés par Southern blot. Au moins $85 \%$ des femmes sont informatives et l'on peut suivre ainsi la co-ségrégation d'un allèle polymorphique révélé par DXS52 et du gène de l'ALD-X. Dans une étude initiale[1l], aucune recombinaison n'a été observée dans 


\section{RÉFÉRENCES}

11. Aubourg PR, Sack GH, Meyers DA, et al. Linkage of adrenoleukodystrophy to a polymorphic DNA probe. Ann Neurol 1987; 21 : 349-52.

12. Nathans J, Thomas D, Hogness DS Molecular genetics of human color vision : The genes encoding blue, green and red pigments. Science 1986 ; 232: 193-202.

13. Nathans J, Piantanida TB, Eddy RL, $e$ al. Molecular genetics of inherited variation in human color vision. Science 1986; 232 203-10.

14. Aubourg PR, Sack GH, Moser HW. Frequent alterations of visual pigment genes in adrenoleukodystrophy: Am J Hum Genet $1988 ; 42: 408-13$

15. Bernheimer $\mathrm{H}$, Budka $\mathrm{H}$, Muller P. Brain tissue immunoglobulins in adrenoleukodystrophy: A comparison with multiple sclerosis and systemic lupus erythematosus. Acta Neuropathol 1983 ; 59 : 95-102.

16. Griffin DE, Moser HW, Mendoza Q, et al. Identification of the inflammatory cells in the central nervous system of patients with adrenoleukodystrophy. Ann Neurol 1985; 18 : 660-4.

17. Kannagi R, Nudelman E, Hakomori SI. Possible role of ceramide in defining structure and function of membrane glycolipids. Proc Natl Acad Sci USA 1982 ; 79 : 3470-4.

18. Rizzo WB, Watkins PA, Phillips MW, et al. Adrenoleukodystrophy : oleic acid lowers fibroblast C22-26 fatty acids. Neurology 1986 ; $36: 357-61$.

19. Moser HW, Tutschka P, Brown III FR, et al. Bone narrow transplant in adrenoleukodystrophy. Neurology $1984 ; 34$ : 1410-7.

20. Hogand B, Lyons K. Gene targeting: getting nearer the mark. Nature 1988; 336 :
7 familles étudiées (65 méioses) permettant de calculer un LOD score* à 13,766 (à $0 \%$ de recombinaison entre Stl4 et l'ALD-X). Cette étude de « linkage » a rendu certaine la localisation du gène de l'ALD-X dans la région $\mathrm{Xq} 2-8$, à proximité des gènes de l'hémophilie $A$, des pigments visuels vert et rouge (daltonisme) et de la myopathie d'Emery Dreyfuss. Plus d'une dizaine de gènes ont été localisés dans cette portion télomérique du chromosome $\mathrm{X}$ et, jusqu'ici, aucune association morbide n'a été observée entre l'ALD-X et une maladie génétique localisée dans cette même région. En particulier, aucun patient atteint d'ALD-X n'est connu pour avoir, soit une hémophilie $A$, soit un déficit en G6PD ou une myopathie d'Emery Dreyfuss. Cependant, une étude initiale de familles d'ALD-X a permis d'établir qu'environ 40 à $50 \%$ des patients étaient daltoniens, c'est-à-dire avaient une difficulté pour voir la couleur rouge ou la couleur verte. Le daltonisme est relativement fréquent puisqu'il survient chez environ $8 \%$ des mâles d'origine «caucasienne » européenne. Parmi ceux-ci, environ la moitié a des anomalies de type deutéranomalie (perception légère- ment défectueuse de la couleur verte) tandis que le reste comprend en proportions approximativement égales des patients deutéranopes (sévère déficit de perception du vert), protanopes (sévère déficit de la perception du rouge) ou des patients ayant des anomalies de type protanomalie (déficit modéré de la perception du rouge). J. Nathans et al. [12, 13] ont cloné les gènes des pigments vert et rouge et montré que les hommes avec une vision des couleurs normale avaient un gène d'un pigment rouge et un ou plusieurs gènes d'un pigment vert ${ }^{* *}$ (figure $4 A$ ). Vollrath et al. ont montré récemment que ces gènes étaient situés en tandem sur une distance d'environ 120 kilobases (kb), le gène du pigment rouge étant situé en position 5' de ce groupe. Les anomalies de perception du rouge et du vert résultent de recombinaisons homologues ou de conversions géniques entre les gènes des pigments vert et rouge (figure $4 \mathrm{~B}$ ). Il en résulte alors des délétions géniques ou des

* LOD score : logarithme décimal du rapport entre la probabilité qu'il existe une liaison entre le locus ALD-X et la sonde DX552 et celle qu'il n'y ait pas de liaison.

** Voir m/s, suppl. au $n^{\circ} 7$, vol. 3, p. 20

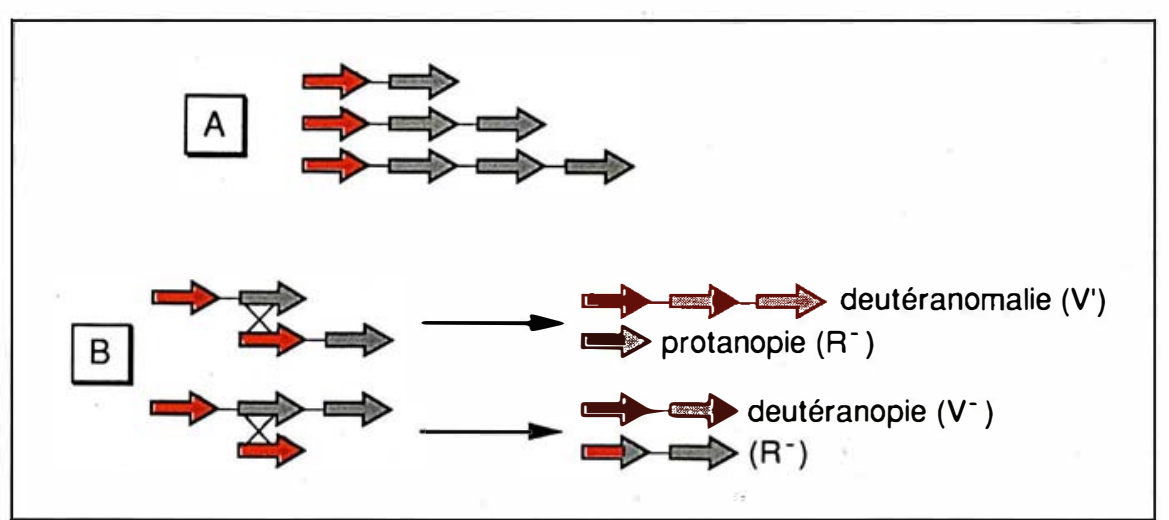

Figure 4. A. Organisation des gènes des pigments visuels rouge et vert chez les hommes ayant une vision des couleurs normale. Tous les individus ont un gène de pigment visuel rouge et un ou plusieurs gènes du pigment visuel vert. B. Réorganisation des gènes des pigments visuels par recombinaison entre les gènes des pigments rouge et vert. Les gènes hybrides sont faits d'une partie $5^{\prime}$ ou $3^{\prime}$ 'des gènes des pigments rouge et vert. Deutéranomalie $\left(V^{\prime}\right)$ : anomalie modérée de perception du vert. Deutéranopie $(V-)$ : sévère anomalie de la perception du vert. Protanopie (R-): sévère anomalie de la perception du rouge. 


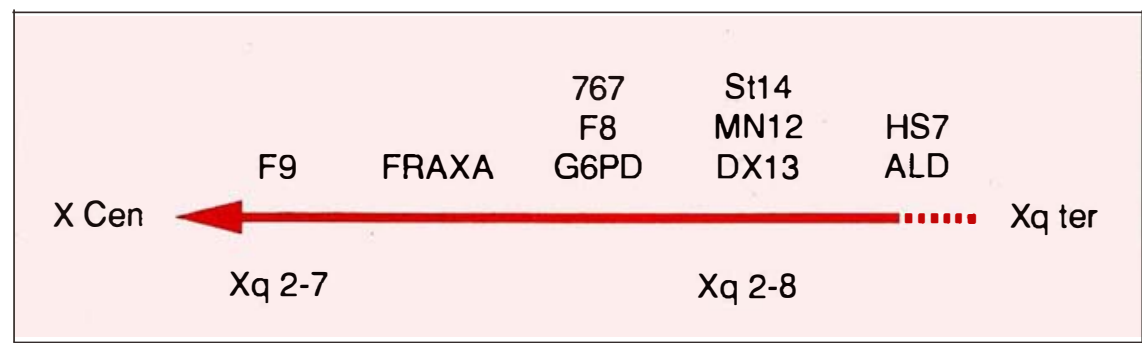

Figure 5. Carte physique de la région Xq2-7 - Xq2-8. F8 et F9: facteurs VIII et IX de la coagulation; FRAXA : site fragile du chromosome $X$ associé à un retard mental; G6PD : glucose-6-phosphate déhydrogénase; HS7: sonde d'ADN complémentaire codant pour le gène du pigment visuel rouge; $A L D-X$ : adrénoleucodystrophie liée à I'X; 767, St14, MN12 et DX13: sondes ADN anonymes révélant un polymorphisme allélique.

gènes hybrides constitués d'une partie d'un gène rouge et d'une partie d'un gène vert (par exemple, l'extrémité 5 ' d'un gène vert et l'extrémité 3' d'un gène rouge ou vice-versa). L'importante homologie entre les séquences codantes et introniques des deux gènes $(98 \%)$ est supposée être responsable de la survenue fréquente de crossing-over inégaux d'où résulte une grande variété de réarrangements. L'incidence relativement élevée de daltonisme démontrée par les études neuro-psychologiques chez les patients atteints d'ALD-X a été confirmée par l'étude de l'ADN de ces patients [14]. Cette observation a immédiatement suggéré que les délétions et réarrangements chromosomiques qui intéressent les gènes des pigments visuels puissent s'étendre dans la région 3' et, présomptivement, intéresser le gène de l'ALD-X lui-même. Dès lors, une nouvelle stratégie peut être développée pour isoler le gène de l'ALD-X. Les techniques d'électrophorèse en champ pulsé permettent en effet de séparer des fragments d'ADN qui peuvent aller jusqu'à $2000 \mathrm{~kb}$. Utilisant des enzymes de restriction qui coupent rarement l'ADN et qui engendrent donc de grands fragments, on peut, avec une sonde ADN, étudier une région qui soit, dans les conditions optimales, étendue $2000 \mathrm{~kb}$ de part et d'autre de cette sonde (figure 5). L'application de cette technique aux patients atteints d'ALD-X avec ou sans daltonisme devrait permettre de taux des AGTLC observés dans le plasma ou les fibroblastes et la sévérité de la maladie. Il est à remarquer par ailleurs que, chez les patients où il existe un déficit apparemment isolé des autres enzymes de la $\beta$ oxydation peroxysomiale (oxydase, protéine bifonctionnelle ou thiolase) [1], la présentation clinique et les lésions neuropathologiques sont différentes, même s'il existe quelques éléments de ressemblance. Il n'est donc pas du tout prouvé que l'accumulation d'AGTLC ait un rôle «toxique en soi. Rappelons enfin que la maladie peut être totalement asymptomatique. D'autres facteurs, génétiques ou non, semblent donc requis pour que se manifeste la maladie. Le seul véritablement identifié pourrait être immunologique. Il existe en effet plusieurs arguments en faveur de cette hypothèse: (1) On retrouve dans le cerveau des patients atteints d'ALD-X une réaction périvasculaire avec accumulation de lymphocytes et de macrophages autour des vaisseaux (figure 6, p. 232). Cela rappelle étrangement ce qui est observé dans la sclérose en plaques. (2) L'aspect d'infiltration cellulaire est compatible avec celui d'une réaction immune contre un antigène du système nerveux central. Outre l'accumulation de cellules mononuclées $(59 \% \mathrm{~T}, 34 \% \mathrm{~T} 4,16 \%$ $\mathrm{T} 8,24 \%$ B et $11 \%$ de monocytes/macrophages), on retrouve une augmentation des IgG et IgA libres dans la zone d'inflammation de la substance blanche [15, 16]. Il n'est toujours pas possible de déterminer si ces phénomènes immunologiques représentent des événements importants, primaires ou secondaires. Ceux-ci pourraient rendre compte de la progression rapide de la maladie, très souvent observée, comme de la fréquente asymétrie des lésions de la substance blanche. Plus important encore, ils pourraient expliquer la variabilité de l'expression phénotypique. Comment relier cependant ces phénomènes immunologiques à l'accumulation d'AGTLC? Une possible explication pourrait être fournie par le fait que la composition en acides gras des gangliosides cérébraux est très anormale dans le cerveau des patients atteints d'ALD-X. Les gangliosides sont considérés comme pouvant jouer un rôle 


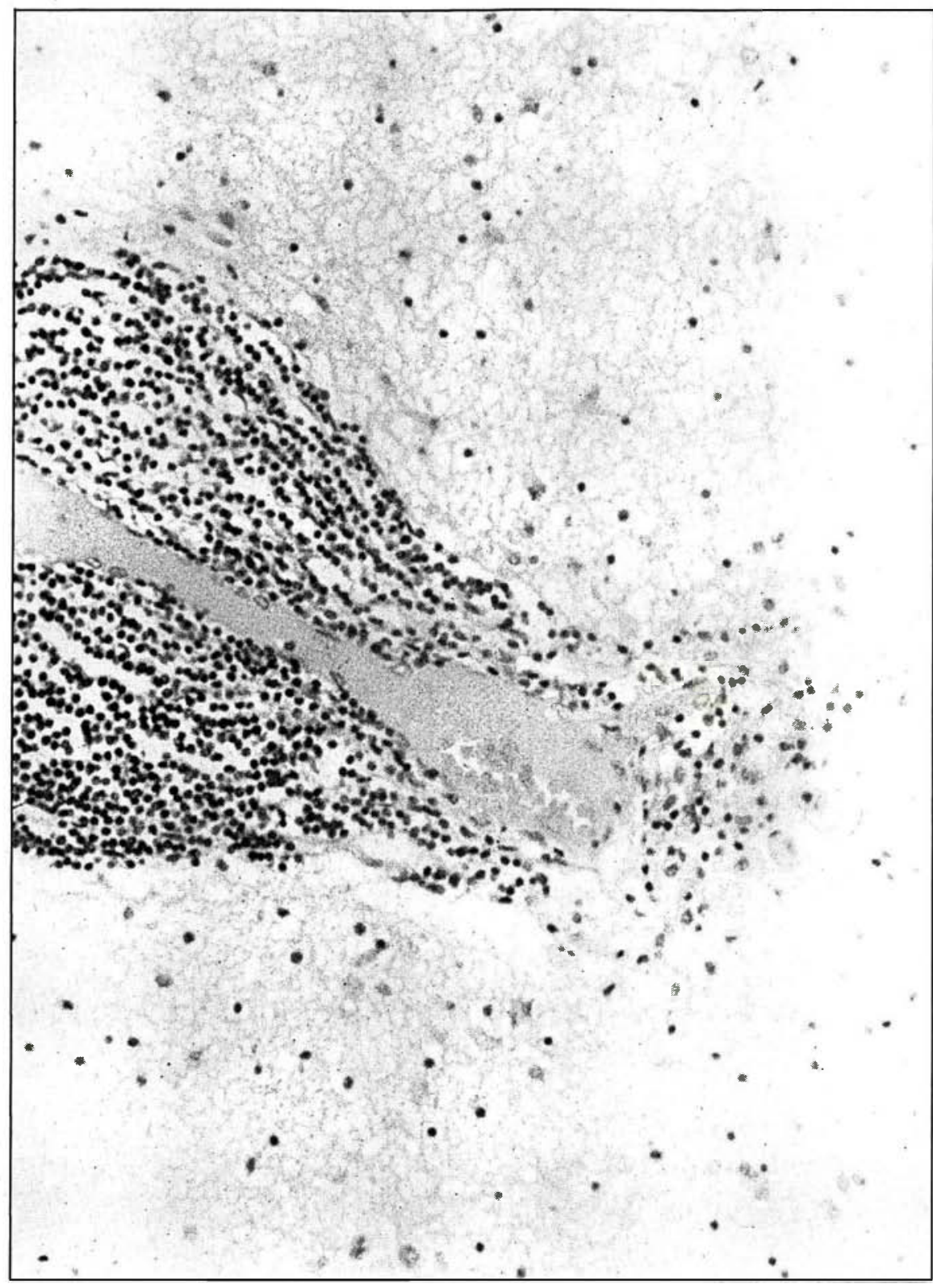

Figure 6. Substance blanche cérébrale: manchons périvasculaires constitués de lymphocytes et de macrophages. (Hématéine-éosine x85.)

important dans les réactions immunologiques et leur composition en acides gras pourrait moduler la réponse immunologique [12].

\section{Perspectives thérapeutiques}

Jusqu'ici, aucune approche théra232
L'acide oléique et encore plus l'acide erucique diminuent considérablement la synthèse de C26:0 dans les fibroblastes de patients atteints d'ALD-X, par un système de compétition avec les enzymes d'élongation à partir du Cl8:0 [18]. Cette approche combinée permet donc, à la fois, de réduire l'apport endogène et exogène des AGTLC et, de fait, l'utilisation d'un tel régime permet de normaliser les taux de C26:0 dans le plasma des patients atteints d'ALD-X. Ce régime n'a malheureusement pas fait la preuve de son efficacité dans les formes déjà neurologiquement évoluées d'ALD cérébrale. Il reste à en évaluer l'effet chez les enfants symptomatiques, mais avant que ne se développent des lésions irréversibles de la substance blanche. Ce traitement semble en revanche beaucoup plus prometteur dans les formes adultes (AMN), où des résultats encourageants ont été déjà obtenus. Une autre approche thérapeutique repose sur la transplantation médullaire. Celle-ci se heurte aux mêmes obstacles que pour les autres maladies dégénératives du SNC. Cependant, pour l'ALD-X, on sait qu'il existe souvent un trouble de la barrière hémato-cérébrale et que les lymphocytes s'accumulent en manchons périvasculaires dans la substance blanche. Si les résultats préliminaires ne sont guère encourageants [19], une des raisons en est la rapide évolution de la maladie, celle-ci une fois déclarée. Il reste donc à déterminer si la transplantation médullaire peut avoir une efficacité lorsqu'elle est faite au tout début de l'évolution de la maladie.

\section{Conclusion}

La pathogénie de l'ALD-X reste encore bien mystérieuse et il n'en existe pas de modèle animal. On peut raisonnablement penser qu'une fois le gène de l'ALD-X isolé, il devrait être possible de créer un modèle animal en mutant le gène normal endogène de l'ALD-X par recombinaison homologue. Cette approche pourrait ainsi permettre d'évaluer plus facilement les différentes tentatives thérapeutiques (greffe médullaire notamment) et d'appréhender les mécanismes responsables de la démyélinisation 


\section{Summary}

Recent advances in adrenoleucodystrophy

X-linked Adrenoleukodystrophy (ALD-X) is a genetic peroxisomal disorder which affects the white matter of the Central Nervous System. The wide phenotypical variation which ranges from the severe and fatal childhood form to persons who remain asymptomatic in adult life suggests that other factors, possibly immunological phenomenons, may play a role in the mechanisms of this disease. Recent advances demonstrated that the underlying biochemical defect, i.e. an impaired capacity to degrade very long chain fatty acids, is due to a deficient peroxisomal very long chain fatty AcylCoA Synthetase. The significantly increased incidence of color pigment gene rearrangements in ALD-X population led to propose that ALD-X and visual pigment genes loci are very closely linked and that aberrant recombination may involve genes adjacent to visual pigment genes and possibly the ALD-X gene itself. Recent therapeutic approaches may offer neurological benefit. 